\title{
Water colliding with oil
}

\author{
David Quéré†
}

Physique et Mécanique des Milieux Hétérogènes, ESPCI,

75005 Paris, France and Departments of Mechanics and Physics, École polytechnique, 91120 Palaiseau, France

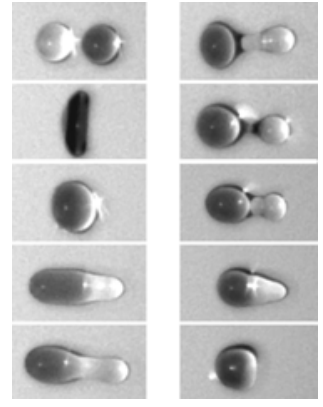

The collision of two liquid drops is both an applied question (in rain formation or combustion, for example) and a beautiful basic situation, where impact involves liquid phases, making this problem worth studying in its own right. In a stimulating paper, Planchette, Lorenceau \& Brenn (J. Fluid Mech., this issue, vol. 702, 2012, pp. 5-25) consider collisions between oil and water, which often lead to water drops protected by a shell of oil. By looking at the deformations during impact, they characterize the dynamical conditions leading to single encapsulation, and derive a criterion for avoiding fragmentation.

Key words: breakup/coalescence, capillary flows, drops

\section{Introduction}

In the domain of interfacial hydrodynamics, impact is a fascinating field of research, founded by Worthington more than one century ago, yet still raising deep and stimulating questions (Worthington 1895; Yarin 2006). The description of a liquid hitting a solid or another liquid forces us to understand a whole range of scales in space and time, and implies the coexistence of interfaces of different natures, geometrical effects of many kinds, and reflections on the possible role of additives in the liquid and even on the role of air, a phase much less passive in the process than we might naively expect (Xu, Zhang \& Nagel 2005). An ink droplet hitting a paper sheet or a raindrop landing on the ground first spreads inertially (so that this phase hardly depends on the nature of the solid), then stops and evolves towards its equilibrium state - a sequence that includes the possibility for the liquid to recoil if solid/liquid interactions are weak. A similar variety of processes is observed for colliding drops: they can merge (minimizing their surface energy) if the impact is slow and well-centred. But air between them does not always have the time to escape during the shock, so that they can also bounce off each other (Tang, Zhang \& Law 2012) - an effect recently exploited to generate levitation of drops on a bath of the same liquid, by shaking the bath at high enough frequency and acceleration (Couder et al. 2005).

At larger velocities, the liquid distorts so dramatically that there is fragmentation of the filaments forming during impact (Ashgriz \& Poo 1990; Jiang, Umemura \& Law 1992). The phase diagram of impacts is of central importance in different fields

$\dagger$ Email address for correspondence: david.quere@espci.fr 
where coalescence is either looked for, or unwanted. For example, coalescence is one of the mechanisms permitting the initiation of rain, when tiny drops (at the scale of $10 \mu \mathrm{m})$ merge in clouds to form large drops $(0.1-1 \mathrm{~mm}$, or even larger) able to fall down despite the wind (Brazier-Smith, Jennings \& Latham 1972). We also try to force the coalescence of droplets with nets to capture traces of humidity in desert landscapes, or with filters to avoid the dissemination of liquid aerosols. Conversely, when we make a spray, we generally try to avoid the recombination of drops, as in combustion chambers where fuel is disintegrated into tiny droplets to make the combustion efficient.

\section{Overview}

An interesting variation on this theme, which mixes the mechanics of impacts with the physics of wetting (owing to the presence of three phases) was considered by Planchette, Lorenceau \& Brenn (2012), who looked at the collision of two drops of different chemical nature (oil and water). Rebound and fragmentation are also observed, but because water and oil do not mix, the product of the collision can be a drop of liquid 1 fully encapsulated by a shell of liquid 2, as sketched in figure 1 $(a)$. The criterion for liquid 2 to coat liquid 1 is derived from a surface energy comparison: a liquid 1 contacting a liquid 2 will become covered by liquid 2 if the surface energy of liquid 1 is larger than the sum of surface energies between liquid 1 and liquid 2, and liquid 2 and air. This criterion can be written in terms of the corresponding surface tensions: $\gamma_{1}>\gamma_{12}+\gamma_{2}$. If it is satisfied, a drop of liquid 1 will be encapsulated by liquid 2 . Note that all the tensions here are measurable, so that the criterion is fully predictive. Water surface tension is $72 \mathrm{mN} \mathrm{m}^{-1}$, while water/oil and oil surface tensions are typically $35 \mathrm{mN} \mathrm{m}^{-1}$ and $20-30 \mathrm{mN} \mathrm{m}^{-1}$ : in most situations, water is encapsulated by oil (owing to its high surface tension). With surfactants, $\gamma_{12}$ can become negligible compared to other tensions, and the criterion of encapsulation simply reduces to $\gamma_{1}>\gamma_{2}$.

Encapsulated drops are noteworthy objects: the liquid core is protected from evaporation or contamination, which permits its transport to a given target without leakage or damage. The shell itself can be solidified by polymerizing the oil, making the process relevant in cosmetics or in pharmaceutical applications. Hence a natural question, raised by Planchette et al. (2012) is: can we make these objects in a dynamical way, taking advantage of the collision, but without breaking up the liquids? Günter Brenn invented a wonderful tool for this kind of study: he designed a drop gun, which shoots at regular intervals calibrated drops (of a fraction of millimetre in size), at a well-defined velocity (in the range of metres per second). This makes it possible to study in a controlled way the collision of liquids of similar or different nature, by shooting two drops against each other, and recording this duel with a camera illuminated by short $(O(\mathrm{~ns}))$ flashes. This is shown in figure $1(b-d)$ (where water was dyed with ink), and compared to what happens when a liquid bounces off a very hot plate (figure 1e), a situation that minimizes dissipation, since it avoids contact between the solid and the liquid.

For all these experiments, typical Weber numbers $(W e)$ are larger than unity. This number, which is written $W e=\rho V^{2} R / \gamma$ (with $\rho$ and $\gamma$ the liquid density and surface tension, $V$ the impact velocity and $R$ the drop radius), compares the kinetic energy of the liquid to its surface energy. Hence $W e>1$ means that significant distortions will occur at impact, as observed in the figure: the liquid first makes a disk, which as it recoils transforms into a cylinder. Strong oscillations follow: interfaces can be viewed 
(a)

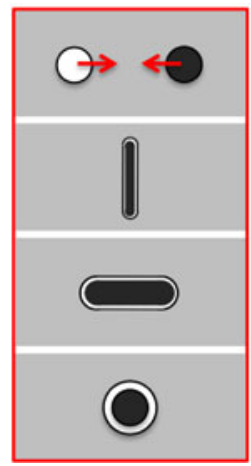

(b)

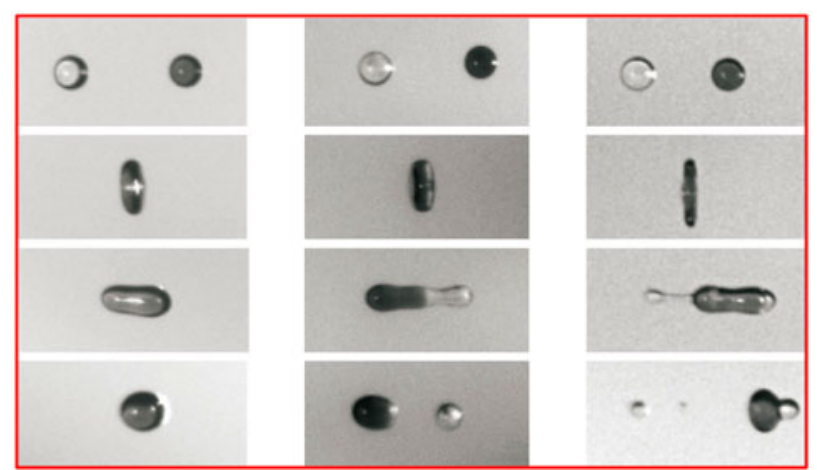

(e)

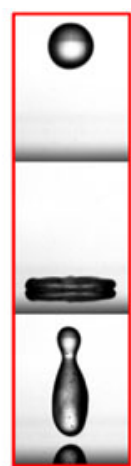

FIGURE 1. (Colour online available at journals.cambridge.org/flm) (a) Sketch of an oil drop (white) impacting a water drop (black): the bi-drop first makes a disk, then transforms into a cylinder, and eventually produces one or several drops. $(b-d)$ Photographs showing oil colliding with water, so that the product of the collision can be a simple water drop encapsulated by oil. Drop radii are $0.1 \mathrm{~mm}$ and the relative velocity at impact is $4 \mathrm{~m} \mathrm{~s}^{-1}$. By varying the viscosity of the oil or water (to which glycerol can be added), one or several drops are made, depending on the length of the transient cylinder (the viscosity of the system decreases from $b$ to $d$ ). Typical Weber numbers are 100. Adapted from Planchette et al. (2012). (e) The impact of a water drop $(R=1.4 \mathrm{~mm})$ at $V=56 \mathrm{~cm} \mathrm{~s}^{-1}$ on a very hot plate, so that the disk and the cylinder are also observed. The corresponding Weber number is 7. Image supplied by Dan Soto.

as springs whose stiffness is surface tension (indeed of dimension Newtons per meter), so that it is possible in these systems to convert kinetic energy into elastic energy, and vice versa. However these springs are somewhat fragile: if cylinders are too long, they break into several pieces, whose number increases with the aspect ratio of the cylinder (see figure $1 c, d$ ).

There are currently debates in the literature about the characteristics of the transient axisymmetric objects forming during an impact (Eggers et al. 2010). Beyond their intrinsic relevance, these questions are also practical: Planchette et al. (2012) found that fragmentation occurs if the aspect ratio of the cylinder is larger than $\pi$, that is, the criterion discovered by Plateau (and discussed by Delaunay and Rayleigh) for the instability of a liquid cylinder. It is remarkable that this 'static' criterion remains valid in a dynamical situation, where internal flows and elasticity arising from the ends of the cylinder might modify it. And if the purpose is to encapsulate liquid 1 by liquid 2 , it is important to understand what determines the aspect ratio of the cylinder to predict whether it will make fragments or not. Let us adopt the simplest point of view, that is, conservation of energy (Chandra \& Avedisian 1991). Assuming that the surface and kinetic energies of the impacting liquid are stored in deformation, first in the disk shape, later in the cylinder shape, we find predictions for the radius $R_{D}$ of the disk, and for the length $L$ of the cylinder. For a drop impacting a hot plate, they are, respectively $R_{D} \simeq R(W e / 3+2)^{1 / 2}$ and $L \simeq 3 R(W e / 6+1)^{2}$. At moderate Weber numbers (e.g. in figure $1 e$, where we have $W e \simeq 7$ ), conservation of energy nicely predicts the radius of the disk (expected from the formula to be about twice the drop radius, as observed), but not the size of the cylinder (predicted to be $\sim 15$ times 
larger than $R$ ). Spreading and recoiling phases of impact are very different from this viewpoint, and these differences largely remain to be explained.

\section{Future}

Energy conservation is not at all relevant at larger We, even in the spreading stage, as seen in figure $1(b-d)$ : typical Weber numbers are 100 so that energy conservation predicts $R_{D}$ of order $5 R$, and $L$ of order $1000 R$ ! In Planchette et al. (2012)'s work, oils have a viscosity $\sim 10$ times larger than water, and glycerol can be added to the water, so that the distortion of the mixed oil/water drop implies a non-negligible dissipation of energy. Planchette et al. use the viscosity of the liquid to prevent the development of the cylinder (in figure 1, the viscosity of the system decreases from figures $1 b$ to $1 d$ ). They also propose nice scaling arguments where this dissipation is taken into account, allowing them to predict convincingly the size of the cylinder - and thus the threshold of fragmentation. As mentioned earlier, these questions are motivated by the applications, and we can find in the engineering literature data and empirical laws to describe these data. It is certainly useful to tackle these problems in a more fundamental way, allowing us to quantitatively understand these complex situations. This of course leads not only to a better control of the process of encapsulation, but also to new solutions: Planchette et al. (2012) report for example that even when fragments are made, oil can be transferred to the different drops of water, so that multiple encapsulated drops are simultaneously achieved. More fundamentally, these experiments make it possible to perform controlled impacts in air, a situation simpler than on a solid, which helps to build a solid ensemble of data on which to base the very active numerical and theoretical research on these subjects.

\section{References}

Ashgriz, N. \& Poo, J. Y. 1990 Coalescence and separation in binary collisions of liquid drops. J. Fluid Mech. 221, 183-204.

BraZier-Smith, P. R., Jennings, S. G. \& Latham, J. 1972 The interaction of falling water drops: coalescence. Proc. R. Soc. Lond. A 326, 393-408.

Chandra, S. \& Avedisian, C. 1991 On the collision of a droplet with a solid surface. Proc. R. Soc. Lond. A 432, 13-41.

Couder, Y., Fort, E., Gautier, C. H. \& Boudaoud, A. 2005 From bouncing to floating: noncoalescence of drops on a fluid bath. Phys. Rev. Lett. 94, 177801.

Eggers, J., Fontelos, M. A., Josserand, C. \& Zaleski, S. 2010 Drop dynamics after impact on a solid wall: theory and simulations. Phys. Fluids 22, 062101.

JiAnG, Y. J., UmemurA, A. \& LAW, C. K. 1992 An experimental investigation on the collision behaviour of hydrocarbon droplets. J. Fluid Mech. 234, 171-190.

Planchette, C., Lorenceau, E. \& Brenn, G. 2012 The onset of fragmentation in binary liquid drop collisions. J. Fluid Mech. 702, 5-25.

TANG, C., ZhANG, P. \& LAW, C. K. 2012 Bouncing, coalescence, and separation in head-on collision of unequal-size droplets. Phys. Fluids 24, 022101.

Worthington, A. M. 1895 The Splash of a Drop. Society for Promoting Christian knowledge.

Xu, L., Zhang, W. W. \& Nagel, S. R. 2005 Drop splashing on a dry smooth surface. Phys. Rev. Lett. 94, 184505.

YARIN, A. L. 2006 Drop impact dynamics: splashing, spreading, receding, bouncing. Annu. Rev. Fluid Mech. 38, 159-192. 